\title{
Soft-decision Star-QAM aided BICM-ID
}

Dandan Liang, Student Member, IEEE, Soon Xin Ng, Senior Member, IEEE, and Lajos Hanzo, Fellow, IEEE

\author{
University of Southampton \\ Communications Research Group \\ Southampton, SO17 1BJ, U.K. \\ E-Mail: $\{$ dl4e08,sxn,lh\}@ecs.soton.ac.uk
}

\begin{abstract}
Differentially detected non-coherent Star Quadrature Amplitude Modulation (Star-QAM) is ideal for low-complexity wireless communications, since it dispenses with high-complexity channel estimation. We conceive softdecision based demodulation for 16-level Star-QAM (16StQAM), which is then invoked for iterative detection aided Bit-Interleaved Coded Modulation (BICM-ID). It is shown that the proposed 16-StQAM based BICM-ID scheme achieves a coding gain of approximately $14 \mathrm{dBs}$ in comparison to the 16-level identical-throughput Differential Phase-Shift Keying (16DPSK) assisted BICM scheme at a bit error ratio of $10^{-6}$.
\end{abstract}

Index Terms-Soft-decision, Iterative detection, Star QAM, BICM-ID, Correlated Rayleigh fading channel

\section{INTRODUCTION}

Coherent detection aided Quadrature Amplitude Modulation (QAM) requires accurate Channel State Information (CSI) in order to avoid false-phase locking, especially when communicating over Rayleigh fading channels [1]-[4]. As a remedy, differentially detected non-coherent Star-QAM was proposed in [5] in order to dispense with high-complexity CSI estimation. More specifically, 16-level Star-QAM (16-StQAM) is based on two concentric 8-level Phase-Shift Keying (8PSK) constellations having two different amplitudes. Differential detection has also been investigated recently in wireless relay networks [6]-[8]. The significance of this low-complexity detection method may be expected to increase in the cooperative communications era, since it might be unrealisitic to expect from a relay station constituted by a cooperating mobile phone to estimate the channel of the link it is relaying [7], [8].

Star-QAM schemes having more than two PSK constellations are also referred to as Differential Amplitude and Phase-Shift Keying (DAPSK) schemes [9], [10]. The authors of [9], [10] have further improved the performance of DAPSK/Star-QAM schemes [9],

The research leading to these results has received funding from the European Union's Seventh Framework Programme ([FP7/20072013]) under grant agreement no [214625]. The financial support of the EPSRC UK in the framework of the IU-ATC and the China-UK project in $4 \mathrm{G}$ wireless communications is also gratefully acknowledged.
[10]. However, despite its attractive performance versus complexity characteristics, soft-decision based demodulation has not been conceived for these Star-QAM and DAPSK schemes. This also implies that without soft-decision based demodulation, the potential power of sophisticated channel coding or coded modulation schemes cannot be fully exploited. Hence, when channel coding is incorporated into Star-QAM as in [5], its performance is far from the channel capacity due to the employment of hard-decision based demodulation. More specifically, powerful channel coding, such as Bit-Interleaved Coded Modulation (BICM) [11], [12] and Iteratively-Detected BICM (BICM-ID) [13], [14] heavily relies on the exploitation of soft-decision based demodulation.

Our novel contribution is that we will first derive the soft-decision demodulation formula for 16-StQAM. Secondly, the performance benefits of using this new formula will be demonstrated in the context of BICM and BICM-ID schemes invoked for communications over correlated Rayleigh fading channels. Note, however, that the proposed soft-decision based 16-StQAM demodulation principles may be readily extended to DAPSK schemes having more than two concentric PSK constellations. This letter is organised as follows. In Section II, the softdecision demodulation of 16-StQAM will be presented. Our results will be discussed in Section III and our conclusions are offered in Section IV.

\section{System MOdel AND ANALYSiS}

Fig. 1 shows the simplified schematic of the proposed 16-StQAM aided BICM-ID scheme. A sequence of 3-bit information symbols is encoded by a rate-3/4 BICM encoder for yielding a sequence of 4-bit coded symbols. The Most Significant Bit (MSB) of the 4bit encoded symbol will be used for selecting the amplitude of the Phase-Shift-Keying (PSK) ring, while the remaining 3 bits will be used for selecting the phase of the complex-valued 16-StQAM symbol $x_{k}$, where the subscript $k$ denotes the symbol index. The BICM-encoded 16-StQAM symbol is corrupted by both 


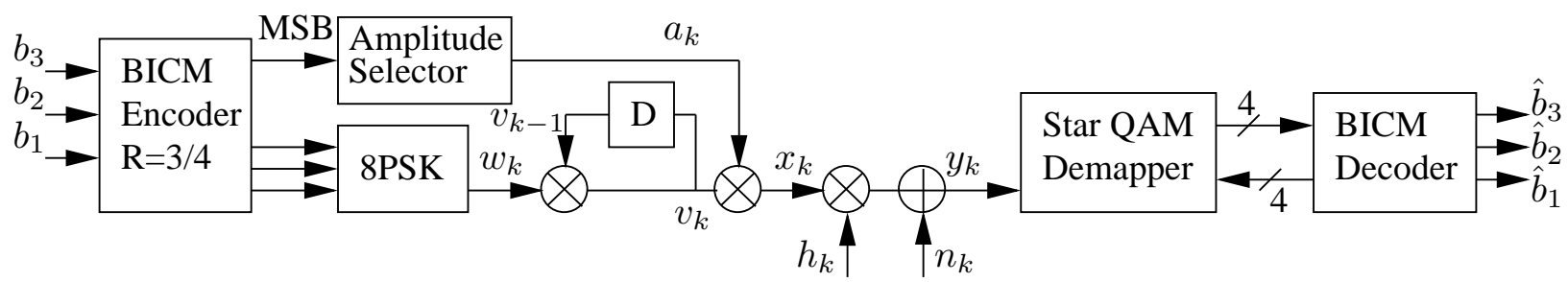

1: The schematic of the 16-StQAM aided BICM-ID scheme, where the parallel bit interleavers between the encoder/decoder and mapper/demapper are not shown for avoiding obfuscating details.

the Rayleigh fading channel $h_{k}$ and the Additive White Gaussian Noise (AWGN) $n_{k}$, when it is transmitted to the receiver, as shown in Fig. 1. Iterative detection is then carried out by exchanging extrinsic information between the 16-StQAM soft demapper and BICM decoder based on the received sequence $\left\{y_{k}\right\}$ without the any need for CSI.

\section{A. Star-QAM Mapper}

As seen in Fig. 1, the 16-StQAM mapper consists of three components, namely the amplitude selector, the 8PSK mapper and a differential encoder. The 8PSK mapper and the differential encoder jointly form a conventional 8-level DPSK (8DPSK) mapper. The MSB of the BICM-encoded symbol, namely $b_{3}$, is used for selecting one of the two possible amplitudes. The remaining 3 bits, namely $b_{2} \quad b_{1} \quad b_{0}$, are used by the 8DPSK mapper. Note that similar to any DPSK scheme, we insert a reference symbol at the beginning of each frame before the 16-StQAM mapper.

1) Amplitude Selection: The MSB, $b_{3}$, is used for selecting the amplitude of the PSK ring, $a_{k}$. The two possible amplitude values are denoted as $a^{(1)}$ and $a^{(2)}$, respectively. When the MSB of the $k$ th BICM-encoded symbol is given by $b_{3}=0$, the amplitude of the PSK ring will remain the same as that of the previous value $a_{k}=a_{k-1}$. The amplitude of the PSK ring will be switched to another value, if $b_{3}=1$. This amplitude selection mechanism may be referred to as 2-level Differential Amplitude Shift Keying (2DASK). After normalisation for maintaining a symbol energy of unity, we have $a^{(1)}=1 / \sqrt{2.5}$ and $a^{(2)}=2 / \sqrt{2.5}$. The amplitude value of the reference symbol is given by $a_{0}=a^{(1)}$.

2) Phase Selection: The $k$ th differentially encoded symbol $v_{k}$ can be expressed as:

$$
v_{k}=v_{k-1} w_{k}
$$

where $x_{k}=\mu\left(b_{2} b_{1} b_{0}\right)$ is the $k$ th 8PSK symbol based on the 8PSK mapping function of $\mu($.$) , while v_{k-1}$ is the $(k-1)$ st 8DPSK symbol and $\left|v_{k}\right|^{2}=1$. The reference symbol for the 8DPSK part is given by $v_{0}=\mu\left(\begin{array}{lll}0 & 0 & 0\end{array}\right)$.
The $k$ th 16-StQAM symbol is then given by:

$$
x_{k}=a_{k} v_{k},
$$

where $a_{k} \in\left\{a^{(1)}, a^{(2)}\right\}$.

\section{B. Star-QAM Soft Demapper}

The soft-decision based 16-StQAM block is placed in front of the BICM decoder of Fig. 1. The $k$ th received symbol may then be written as:

$$
y_{k}=h_{k} x_{k}+n_{k}=h_{k} a_{k} v_{k}+n_{k},
$$

where $h_{k}$ is the Rayleigh fading channel's coefficient, while $n_{k}$ represents the AWGN having a variance of $N_{0} / 2$ per dimension. Assuming a slow Rayleigh fading channel, where $h_{k} \approx h_{k-1}$, we can rewrite (3) using (1) as:

$$
\begin{aligned}
y_{k} & =h_{k-1} a_{k} v_{k-1} w_{k}+n_{k}, \\
& =\frac{a_{k}}{a_{k-1}}\left(y_{k-1}-n_{k-1}\right) w_{k}+n_{k}, \\
& =p_{k} y_{k-1} w_{k}+\tilde{n}_{k},
\end{aligned}
$$

where $p_{k}=\frac{a_{k}}{a_{k-1}}$ is the ratio of the $k$ th and $(k-1)$ st amplitudes, while $\tilde{n}_{k}=-\frac{a_{k}}{a_{k-1}} n_{k-1} w_{k}+n_{k}$ is the effective noise.

1) Amplitude Detection: Three amplitude ratios can be derived from the two PSK ring amplitudes of 16StQAM as follows:

$$
p_{k}=\left\{\begin{array}{l}
R_{0}=\frac{a^{(1)}}{a^{(1)}} \text { or } \frac{a^{(2)}}{a^{(2)}}=1 \\
R_{1}=\frac{a^{(1)}}{a^{(2)}} \\
R_{2}=\frac{a^{(2)}}{a^{(1)}} .
\end{array}\right.
$$

When the noise power is low, the amplitude ratio $p_{k}$ may be approximated as:

$$
\begin{aligned}
\frac{\left|y_{k}\right|}{\left|y_{k-1}\right|} & =\frac{\left|h_{k} a_{k} v_{k}+n_{k}\right|}{\left|h_{k-1} a_{k-1} v_{k-1}+n_{k-1}\right|}, \\
& \approx \frac{\left|a_{k}\right|}{\left|a_{k-1}\right|}, \\
& \approx p_{k} .
\end{aligned}
$$


Fig. 2 shows the Probability Density Function (PDF) of the received signal amplitude ratios $\frac{\left|y_{k}\right|}{\left|y_{k-1}\right|}$. It becomes plausible from Fig. 2 that the PDF peak, which is characteristic of each amplitude ratio experiences a different noise variance, although all the 16-StQAM symbols experience the same AWGN at the same $E_{b} / N_{0}$ value of $25 \mathrm{~dB}$.

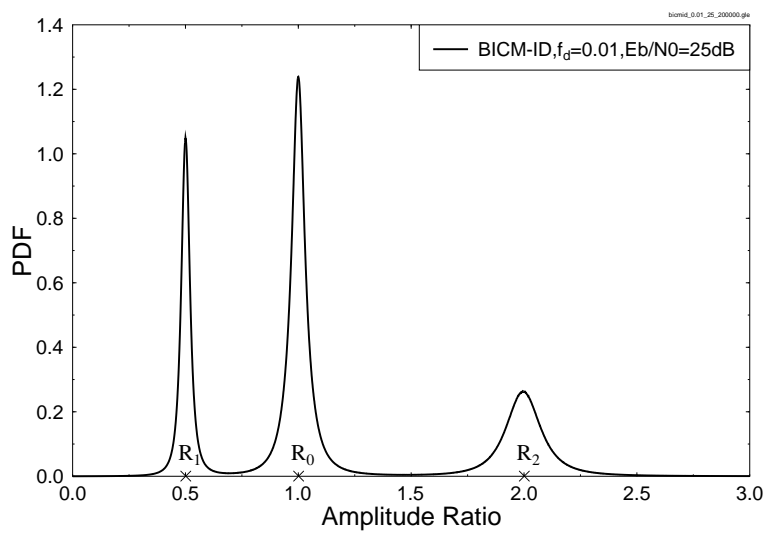

2: The PDF of the received signal amplitude ratios of 16StQAM $\frac{\left|y_{k}\right|}{\left|y_{k-1}\right|}$ based on (6), when communicating over correlated Rayleigh fading channels having an $E_{b} / N_{0}$ of $25 \mathrm{~dB}$.

2) Probability Computation: The effective noise variance of $\tilde{n}_{k}$ in (4) depends on the amplitude ratio used at time instant $k$, which can be computed as:

$$
\widetilde{N}_{0}=N_{0}+\left|p_{k}\right|^{2}\left|w_{k}\right|^{2} N_{0}=N_{0}\left(1+\left|p_{k}\right|^{2}\right) \text {, }
$$

where $\widetilde{N}_{0}=2 N_{0}=N_{0}^{(0)}$ if $b_{3}=0$, while $\tilde{N}_{0}=$ $\left(1+R_{1}^{2}\right) N_{0}=N_{0}^{(1)}$ or $\widetilde{N}_{0}=\left(1+R_{2}^{2}\right) N_{0}=N_{0}^{(2)}$ for $b_{3}=1$. Based on (4) we can express the probability of receiving $y_{k}$ conditioned on the transmission of $b_{0}, b_{1}$, $b_{2}$ and $b_{3}$ as follows:

$$
\begin{aligned}
P\left(y_{k} \mid w^{(m)}, b_{3}=0\right)= & \frac{1}{\pi N_{0}^{(0)}} e^{\frac{-\left|y_{k}-y_{k-1} R_{0} w^{(m)}\right|^{2}}{N_{0}^{(0)}}} \\
P\left(y_{k} \mid w^{(m)}, b_{3}=1\right)= & \frac{1}{\pi N_{0}^{(1)}} e^{\frac{-\left|y_{k}-y_{k-1} R_{1} w^{(m)}\right|^{2}}{N_{0}^{(1)}}}+ \\
& \frac{1}{\pi N_{0}^{(2)}} e^{\frac{-\left|y_{k}-y_{k-1} R_{2} w^{(m)}\right|^{2}}{N_{0}^{(2)}}}
\end{aligned}
$$

where $w^{(m)}=\mu\left(\begin{array}{lll}b_{2} & b_{1} & b_{0}\end{array}\right)$ and $\mu$ is the conventional 8PSK mapping function. However, when the a priori bit probabilities $P^{a}\left(b_{i}\right)$ become available from the BICM decoder, the extrinsic bit probability that can be gleaned from the 16-StQAM demapper becomes:

$$
\begin{aligned}
& P^{e}\left(b_{i}=b\right)= \\
& \sum_{w^{(m)} \in \chi(i, b)}\left(P\left(y_{k} \mid w^{(m)}, b_{3}=0\right)+P\left(y_{k} \mid w^{(m)}, b_{3}=1\right)\right) \prod_{\substack{j=0 \\
j \neq i}}^{3} P^{a}\left(b_{j}\right),
\end{aligned}
$$$$
\text { for } i \in\{0,1,2\}, \quad b \in\{0,1\},
$$

where $b_{i}$ denotes the $i$ th coded bit of the symbol and $\chi(i, b)$ is the set of constellation points having the $i$ th bit set to $b$. The extrinsic bit probability of the MSB may be formulated as:

$$
P^{e}\left(b_{3}=b\right)=\sum_{w^{(m)}}^{\text {all }} P\left(y_{k} \mid w^{(m)}, b_{3}=b\right) \prod_{j=0}^{2} P^{a}\left(b_{j}\right),
$$

where the summation term considers all possible 8PSK constellation points, because the MSB $b_{3}$ influences only the amplitude selection. The extrinsic bit probabilities can then be employed for generating the Log-Likelihood Ratios (LLRs) [15] of all BICM-coded bits, which are then fed back to the BICM decoder.

\section{Simulation Results}

Monte-Carlo simulations have been performed for characterising the proposed soft-decision based 16StQAM demodulation technique in the context of BICM and BICM-ID coding schemes. The simulation parameters are shown in Table I.

\begin{tabular}{|l|c|c|}
\hline $\begin{array}{l}\text { Coded } \\
\text { Modulation }\end{array}$ & BICM & BICM-ID \\
\hline Modulation Scheme & $\begin{array}{l}\text { 16-StQAM, 16PSK } \\
\text { 16QAM, 16DPSK }\end{array}$ & 16-StQAM \\
\hline Mapper type & Gray-labelled & Set-Partitioned \\
\hline $\begin{array}{l}\text { Number of } \\
\text { iterations }\end{array}$ & 1 & $1,2,4$ \\
\hline Code Rate & \multicolumn{2}{|c|}{$3 / 4$} \\
\hline Code Memory & \multicolumn{2}{|c|}{3} \\
\hline Code Polynomial (octal) & A $14444 ; 0624 ; 0256]$ \\
\hline Decoder type & \multicolumn{2}{|c|}{ Approximate Log-MAP } \\
\hline Symbols per frame & \multicolumn{2}{|c|}{1,200} \\
\hline Number of frames & \multicolumn{2}{|c|}{ Correlated Rayleigh channel } \\
\hline Channel & \\
\hline $\begin{array}{l}\text { Normalised Doppler } \\
\text { Frequency }\left(f_{d}\right)\end{array}$ & \multicolumn{2}{|c}{} \\
\hline
\end{tabular}

I: Simulation parameters. Note that we declare 'an iteration' being completed when both the demapper and decoder were activated once.

Fig. 3 portrays the $E_{b} / N_{0}$ performance of the 16DPSK aided BICM, 16-StQAM assisted BICM, 16PSK aided BICM, 16QAM BICM and 16-StQAM based BICMID schemes, when communicating over correlated Rayleigh fading channels. Solid lines are used for illustrating the performance of Gray-labelled BICM, while the dotted lines represent the Set-Partitioning (SP) based 16-StQAM BICM-ID. As seen from Fig. 3, the 16DPSK-BICM scheme suffers from a high BER 


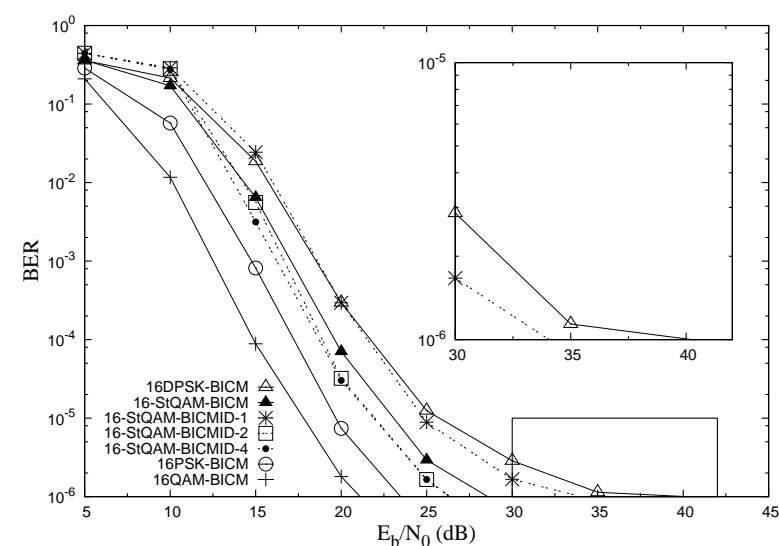

3: BER versus $E_{b} / N_{0}$ performance of the $16 \mathrm{DPSK}-$ BICM, 16-StQAM-BICM, 16-StQAM-BICM-ID, 16PSK-BICM and 16QAM-BICM schemes. The simulation parameters are shown in Table I

floor, since the minimum Euclidean distance of a 16point constellation ring is lower than that of the classic square 16QAM or 16-StQAM schemes. The 16-StQAMBICM scheme outperforms the 16DPSK-BICM scheme by approximately $12 \mathrm{dBs}$ at a BER of $10^{-6}$. The coherently detected 16QAM-BICM and 16PSK-BICM are considered here as our benchmark schemes, while assuming perfect CSI. During the first iteration, the SP-based 16-StQAM-BICM-ID scheme performs worse than the Gray-labelled 16-StQAM-BICM, since the SP-based mapper has a lower minimum Euclidean distance compared to that of the Gray-label-based mapper. Note that both the 16-StQAM-BICM-ID and 16-StQAM-BICM schemes use the bit-probabilities of (9) and (10) during the first iteration. However, after the second iteration the 16-StQAM-BICM-ID outperforms the non-iterative 16-StQAM-BICM by approximately $2 \mathrm{~dB}$ with the aid the extrinsic bit-probabilities of (11) and (12).

\section{CONCLUSIONS}

In this paper, soft-decision based demodulation was conceived for 16-StQAM in order to enable the employment of power-efficient channel codes and coded modulation. The performance of soft-decision 16-StQAM assisted BICM and BICM-ID schemes was investigated, when communicating over correlated Rayleigh fading channels. The proposed soft-decision aided 16-StQAM demodulation techniques can be extended for assisting DAPSK schemes having more than two PSK constellations.

\section{REFERENCES}

[1] E. Issman and W. Webb, "Carrier recovery for 16-level QAM in mobile radio," IEE colloquium on multi-level modulation, pp. 9/1
- 9/8, March 1990.

[2] L. Hanzo, S. X. Ng, T. Keller, and W. Webb, Quadrature Amplitude Modulation: From Basics to Adaptive Trellis-Coded, Turbo-Equalised and Space-Time Coded OFDM, CDMA and MC-CDMA Systems Digital Communications, 2nd ed. WileyIEEE Press, 2004.

[3] L. Chen, H. Kusaka, and M. Kominami, "Blind phase recovery in QAM communication systems using higher order statistics," Signal Processing Letters, IEEE, vol. 3, no. 5, pp. 147 -149, may. 1996.

[4] Y. Wang and E. Serpedin, "A class of blind phase recovery techniques for higher order QAM modulations: estimators and bounds," Signal Processing Letters, IEEE, vol. 9, no. 10, pp. 301 - 304, oct. 2002.

[5] W. Webb, L. Hanzo, and R. Steele, "Bandwidth-efficient QAM schemes for Rayleigh-fading channels," IEE Proceedings, vol. 138, no. 3, pp. 169-175, June 1991 .

[6] Y. Jing and H. Jafarkhani, "Distributed differential space-time coding for wireless relay networks," Communications, IEEE Transactions on, vol. 56, no. 7, pp. 1092 - 1100, July 2008.

[7] L. Wang and L. Hanzo, "The amplify-and-forward cooperative uplink using multiple-symbol differential sphere-detection," IEEE Signal Processing Letters, vol. 16, no. 10, pp. 913 - 916, 2009.

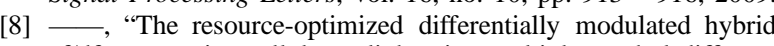
af/df cooperative cellular uplink using multiple-symbol differential sphere detection," IEEE Signal Processing Letters, vol. 16, no. 11 , pp. $965-968,2009$.

[9] C.-D. Chung, "Differentially amplitude and phase-encoded QAM for the correlated Rayleigh-fading channel with diversity reception," IEEE Transactions on Communications, vol. 45, no. 3, pp. 309 - 321, March 1997.

[10] Y. Ma, Q. T. Zhang, R. Schober and S. Pasupathy, "Diversity reception of DAPSK over generalized fading channels," IEEE Transactions on Wireless Communications, vol. 4, no. 4, pp. 1834 - 1846, July 2005.

[11] E. Zehavi, "8-PSK trellis codes for a Rayleigh fading channel," IEEE Transactions on Communications, vol. 40, pp. 873-883, May 1992.

[12] G. Caire and G. Taricco and E. Biglieri, "Bit-Interleaved Coded Modulation," IEEE Transactions on Information Theory, vol. 44, no. 3, pp. 927-946, May 1998.

[13] X. Li and J. A. Ritcey, "Bit-interleaved coded modulation with iterative decoding using soft feedback," IEE Electronics Letters, vol. 34, pp. 942-943, May 1998.

[14] N. Tran, H. Nguyen, and T. Le-Ngoc, "Multidimensional subcarrier mapping for bit-interleaved coded ofdm with iterative decoding," Signal Processing, IEEE Transactions on, vol. 55 no. 12 , pp. $5772-5781$, dec. 2007.

[15] L. Hanzo, T. H. Liew, B. L. Yeap, Turbo Coding, Turbo Equalisation and Space-Time Coding for Transmission over Fading Channels. Wiley-IEEE Press, 2002. 